\title{
An algorithm for detection of Tuberculosis bacilli in Ziehl-Neelsen sputum smear images
}

\author{
Christian del Carpio ${ }^{1}$, Erwin Dianderas ${ }^{2}$, Mirko Zimic ${ }^{3}$, Patricia Sheen ${ }^{4}$, Jorge Coronel ${ }^{5}$, \\ Roberto Lavarello ${ }^{6}$, Guillermo Kemper ${ }^{7}$ \\ 1,2,7 Faculty of Electrical and Electronic Engineering, Universidad Nacional de Ingeniería, Lima 15102, Perú \\ ${ }^{3,4}$ Laboratory of Bioinformatics and Molecular Biology - Faculty of Sciences- Universidad Peruana Cayetano Heredia, \\ Lima 15102, Peru \\ ${ }^{5}$ Tuberculosis Laboratory - Universidad Peruana Cayetano Heredia, Lima 15102, Peru \\ ${ }^{6}$ Pontificia Universidad Católica del Perú, Lima 15088, Perú
}

\section{Article Info}

Article history:

Received Feb 28, 2019

Revised Mar 31, 2019

Accepted Apr 3, 2019

\section{Keywords:}

Baciloscopy

Image processing

Sputum smear

Tuberculosis

Ziehl-Neelsen

\begin{abstract}
This work proposes an algorithm oriented to the detection of tuberculosis bacilli in digital images of sputum samples, inked with the Ziehl Neelsen method and prepared with the direct, pellet and diluted pellet methods. The algorithm aims at automating the optical analysis of bacilli count and the calculation of the concentration level. Several algorithms have been proposed in the literature with the same objective, however, in no case is the performance in sensitivity and specificity evaluated for the 3 preparation methods. The proposed algorithm improves the contrast of the colors of interest, then thresholds the image and segments by labeling the objects of interest (bacilli). Each object then has its geometrical descriptors and photometric descriptors. With all this, a characteristic vector is formed, which are used in the training and classification process of an SVM. For the training 225 images obtained by the 3 preparation methods were used. The proposed algorithm reached, for the direct method, a sensitivity level of $93.67 \%$ and a specificity level of $89.23 \%$. In the case of the Pellet method, a sensitivity of $92.13 \%$ and a specificity of $82.58 \%$ was obtained, while for diluted Pellet the sensitivity was $92.81 \%$ and the specificity $83.61 \%$.
\end{abstract}

Copyright (C) 2019 Institute of Advanced Engineering and Science. All rights reserved.

\section{Corresponding Author:}

Guillermo Kemper,

Faculty of Electrical and Electronic Engineering,

Universidad Nacional de Ingeniería,

Lima 15102, Peru.

Email: guillermo.kemper@gmail.com

\section{INTRODUCTION}

The automation of the optical analysis of sputum samples inked with the Ziehl-Neelsen method [1] is still quite required in different medical centers in different parts of the world that use microscopy to evaluate the samples and determine the presence of tuberculosis bacilli and their respective concentration $[2,3]$. The automation process seeks to reduce the time of obtaining results and improve the precision with respect to that obtained by visual inspection by a medical specialist [4].

The evaluation of the detection algorithms is done through the parameters of sensitivity and specificity. These parameters allow to compare the results of the algorithm with those obtained by coincidence of more than one specialist or observer. Sensitivity measures the algorithm's ability to correctly detect objects that are actually bacilli, while specificity measures the ability to correctly detect objects that are not bacilli. Both parameters are expressed as a percentage, and the closer to $100 \%$ they are, the greater the performance of the algorithm will be. Several computational algorithms have been proposed in the literature in order to be able to execute the analysis of the samples and provide results in the same scheme that a specialist would do. 
Costa et al. [5] were the first to propose an automatic TB bacilli identification method for images acquired with bright field microscopy based on image processing techniques. In their work, they used images that resulted from subtracting the R component minus the $\mathrm{G}$ component $(\mathrm{R}-\mathrm{G})$ from the RGB color format. Their analysis shows that bacilli are more visible in images (R-G) than in RGB images or HSI images. To segment the bacilli, the authors used the histogram from the R-G image and a threshold value was calculated. Morphological and size filters were subsequently applied to remove artifacts from the segmented images. The results presented in the paper were promising; however, his method showed a low sensitivity $(76.65 \%)$.

A Bayesian segmentation method was used in Sadaphal et al. [6] to segment the bacilli. Once the object of interest was segmented, the objects were classified as TB bacilli or non-TB bacilli according to their shape characteristics. The authors used shape descriptors such as axis ratio and eccentricity. However, they do not indicate results obtained from the application of their proposal.

Osman et al [7] used a single-layer feed-forward neural network trained by the Extreme Learning Machine (ELM) technique. The neural network genetic algorithm (GA-NN) approach was used in [8] and the single-layer compact occult feed-forward neural network (C-SLFN) trained by an improved ELM method was applied in [9]. Although the above methods produced acceptable results, the efficacy of the methods was not demonstrated with images with different backgrounds. The nature of the background is an important factor affecting the performance of most autofocus and TB detection methods. Thus, the best result was obtained in [8] where accuracy of test classification was achieved ranging from $84.64 \%$ to $88.21 \%$ with an average of $86.32 \%$.

In 2010, Zhai et al. [10] proposed an automatic image capture system and the application of methods for the detection of TB bacilli. An automatic approach was used for the capture system, using a moving plate that can be moved in the $\mathrm{x}, \mathrm{y}$ and $\mathrm{z}$ coordinates with the help of a stepper motor. The authors performed twostage segmentation such as coarse and fine segmentation by converting RGB images into HSV and CIE Lab color spaces. Its detection process for each sputum sample takes from 3 to 5 minutes. The results obtained for $95 \%$ of the images processed are more than $80 \%$ accurate. The images with an accuracy between $81 \%$ and $90 \%$ represent $72 \%$ of the 100 processed images.

CostaFilho et al. developed a neural network approach in [11]. This method initially classifies the image into a low-density or high-density background image using the image hue component of the HSI color model and a set of color characteristics for segmentation and classification. For high-density background images, the color ratio was used as an additional parameter for efficient sorting. The overall sensitivity obtained was $91.53 \%$, while the false detection was $8.51 \%$.

Again Costa et al. [12] provides an image database to test for the detection of tuberculosis bacilli in 2014. The database consists of two parts: an autofocus database with 1200 images and a segmentation and classification database with 120 images. The images with different backgrounds in the database will help conduct extensive experiments to see how existing and new methods work under various conditions. These marked objects could be used as gold standard to calculate the accuracy, sensitivity and specificity of bacillus recognition. In this case no sensitivity and specificity results of bacillus detection are presented.

Ayas et al. [13] proposed a segmentation and classification method based on the randon forest (RF) learning method. The experimental analysis proposed by the method performs better than other conventional classification methods, such as artificial neural networks (ANNs) and support vector machines (SVMs). Although many methods were presented for segmentation of TB bacilli from conventional microscopic images, a common drawback of most methods is their inability to classify superimposed bacilli as true bacilli. The sensitivity and specificity of the proposed classifier are greater than 75.77 and $96.97 \%$ for pixel segmentation, respectively. It is also mentioned that sensitivity is increased to more than $93 \%$ when staining is performed according to the procedure.

Panicker et al.[14] in 2018, present an automatic method for the detection of Tuberculosis (TB) bacilli by image binarization and subsequent classification of regions detected using a convolutional neural network. They evaluated the algorithm using a data set of 22 images with different backgrounds (high density and low density images). The experimental results show that the proposed algorithm achieves $78.4 \%$ accuracy and 86.76\% F-score for $\mathrm{TB}$ detection. In the present work, although the image processing and classification techniques are well known, they have been adapted and improved aiming to obtain the best results for each preparation method in Ziel Neelsen bacilocopy: direct method, Pellet and diluted Pellet.

\section{RESEARCH METHOD}

Figure 1 shows the block diagram of the proposed algorithm. The image of the sputum sample prepared by a specific method (direct, pellet or diluted pellet) is digitized through a digital camera installed in an optical microscope. The format of the acquired image is RGB (Red-Green-Blue). The obtained image is

An algorithm for detection of Tuberculosis bacilli in Ziehl-Neelsen sputum smear ... (Christian del Carpio) 
treated by the different stages of the processing algorithm that are described below. Sample preparation methods are also described.

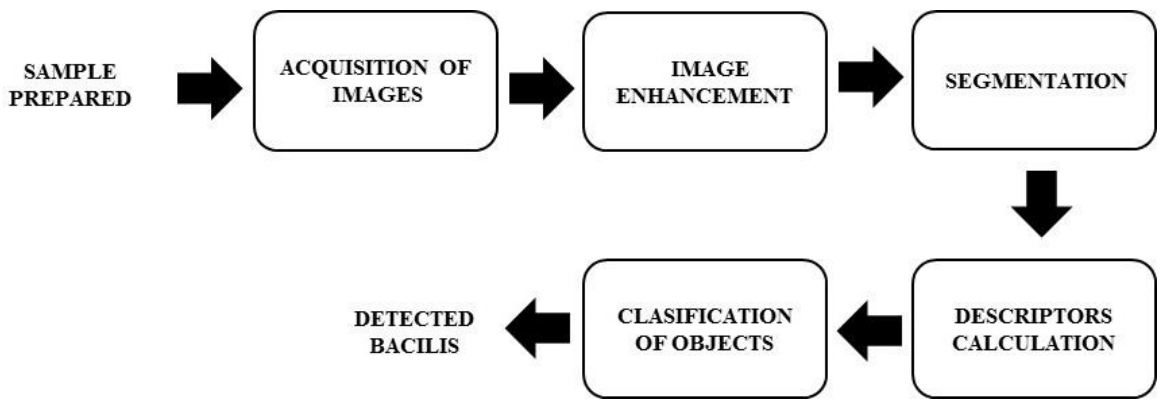

Figure 1. Proposed method flowchart

\subsection{Sample preparation}

Three types of $\mathrm{ZN}$ inked sputum baciloscopy are used in this paper. First, there is direct smear staining, which consists of staining sputum samples on a slide as shown in Figure 2(a) without treatment to purify and concentrate them refer Figure 2(b). On the other hand, concentrated baciloscopy or pellet is the staining of the samples with decontamination and concentration treatment refer Figure 2(c). Finally, dilute baciloscopy or dilute pellet is the staining of samples from the concentrated but diluted sample as shown in Figure 2(d).

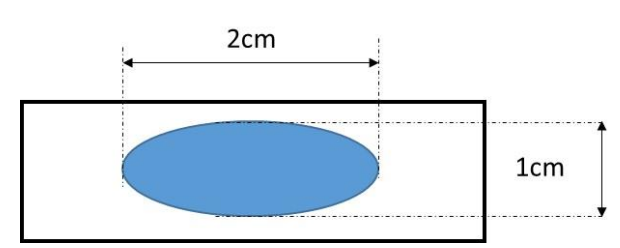

(a)

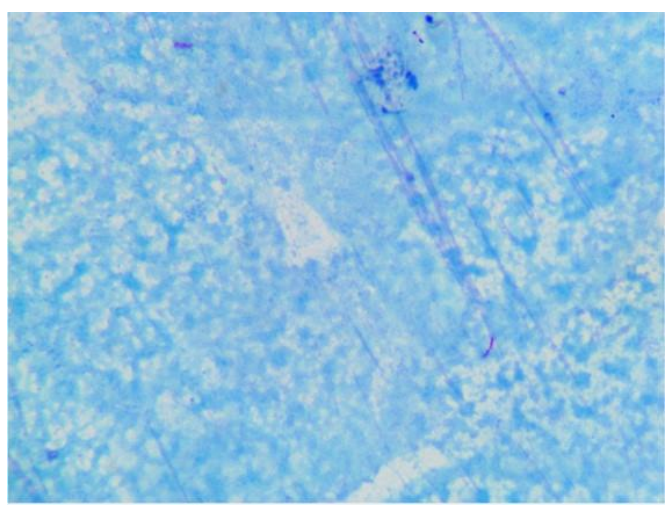

(c)

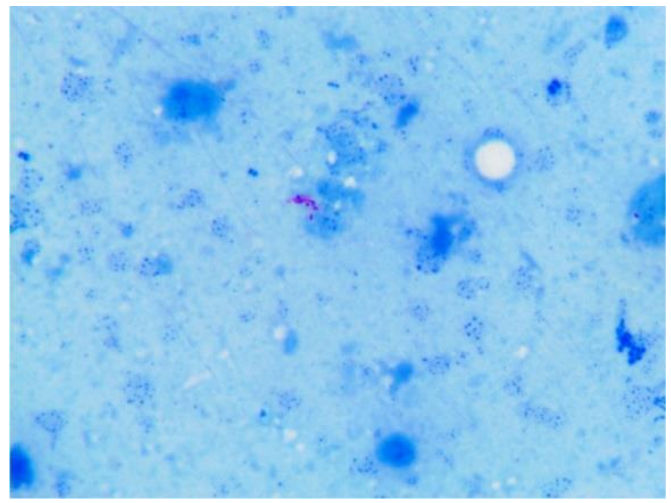

(b)

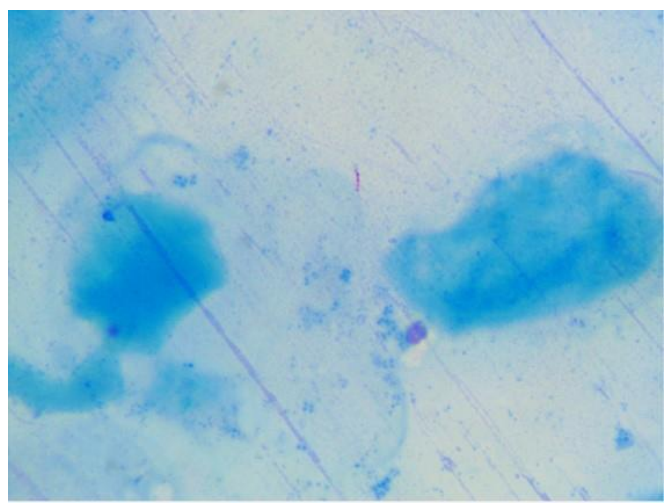

(d)

Figure 2. Sample preparation methods: (a) Object carrier plate, (b) ZN baciloscopy image by direct method, (c) ZN baciloscopy image by pellet method, (d) ZN baciloscopy image by diluted pellet method 


\subsection{Acquisition of images}

All samples, under the three preparation methods, were obtained using a MiniVID microscope camera with CMOS sensor. The images were acquired in RGB color model ( 8 bits per band) with spatial resolution of $2592 \times 1944$ pixels and stored in bitmap format (.bmp). This RGB image is made up of component $R$ expressed as $R(x, y)$, component $G$ expressed as $\mathrm{G}(x, y)$ and component $B$ expressed as $\mathrm{B}(x, y)$.

\subsection{Image enhancement}

The image enhancement process consists of two parts. The first part is to apply consecutively an enhancement filter and a contrast adjustment process. This will allow the edges of the bacilli to be more intense. To apply the enhancement filter, you must first convert from the RGB color model to the CIE color model $L^{*} a^{*} b^{*}[15]$. The components of this image are the $L$ component expressed as $L(x, y)$ and the components $\mathrm{a}^{*}$ and $\mathrm{b}^{*}$ defined as $a^{*}(x, y)$ and $b^{*}(x, y)$ respectively. To perform the conversion (1), (2), (3) and (4) are applied.

$$
\begin{aligned}
& {\left[\begin{array}{l}
X(x, y) \\
Y(x, y) \\
Z(x, y)
\end{array}\right]=\left[\begin{array}{lll}
0.412453 & 0.357582 & 0.180423 \\
0.212671 & 0.715160 & 0.072169 \\
0.019334 & 0.119193 & 0.950227
\end{array}\right]\left[\begin{array}{l}
R(x, y) \\
G(x, y) \\
B(x, y)
\end{array}\right]} \\
& L(x, y)= \begin{cases}116 \cdot\left(\frac{Y(x, y)}{Y_{0}}\right)^{\frac{1}{3}}-16, & \frac{Y(x, y)}{Y_{0}}>0.008856 \\
903.3 \cdot\left(\frac{Y(x, y)}{Y_{0}}\right), \text { otherwise }\end{cases} \\
& a^{*}(x, y)=500 \cdot\left[\left(\frac{X(x, y)}{X_{0}}\right)^{\frac{1}{3}}-\left(\frac{Y(x, y)}{Y_{0}}\right)^{\frac{1}{3}}\right] \\
& b^{*}(x, y)=200 \cdot\left[\left(\frac{Y(x, y)}{Y_{0}}\right)^{\frac{1}{3}}-\left(\frac{Z(x, y)}{Z_{0}}\right)^{\frac{1}{3}}\right]
\end{aligned}
$$

where $X_{0}, Y_{0}, Z_{0}$ tristimulus values of the reference white [16].

Highlight filtering on a color image is applied only on the $L$ component; the $\mathrm{a}^{*}$ and $\mathrm{b}^{*}$ components are not modified. The $L(x, y)$ image is then filtered with a 9x9 MG Gaussian mask and standard deviation $(\sigma)$ of 2 , which is obtained by (5). The image resulting from the Gaussian filtering process is the image $L_{f}(x, y)$, which is obtained by $(6)$.

$$
\begin{aligned}
& M G(i+4, j+4)=\frac{1}{23.99} \sum_{i=-4}^{4} \sum_{j=-4}^{4} e^{-\left(\frac{i^{2}+j^{2}}{2 \sigma^{2}}\right)} \\
& L_{f}(x, y)=L(x, y) * M G(x, y)
\end{aligned}
$$

Applying (7) you get $L^{\prime}(x, y)$, which is the $L$ component filtered with the enhancement filter.

$$
L^{\prime}(x, y)=2.5 \cdot L(x, y)-1.5 \cdot L_{f}(x, y)
$$

Finally, we return to the RGB color model but with the $L$ component modified, expressed as $L^{\prime}(x, y)$, obtaining in RGB color model a new image. The result of the enhancement filtering process is shown in Figure 3(b). After applying the enhancement filter, a contrast enhancement is applied. Once the enhanced RGB image is obtained, its three primary color components are separated from the image. That is, component $R$ expressed as $R^{\prime}(x, y)$, component $G$ expressed as $G^{\prime}(x, y)$ and component $B$ expressed as $B^{\prime}(x, y)$. As can be seen in Figure 4, the most salient information regarding the bacilli can be found in $R$ and $G$ components of the enhanced RGB image. 


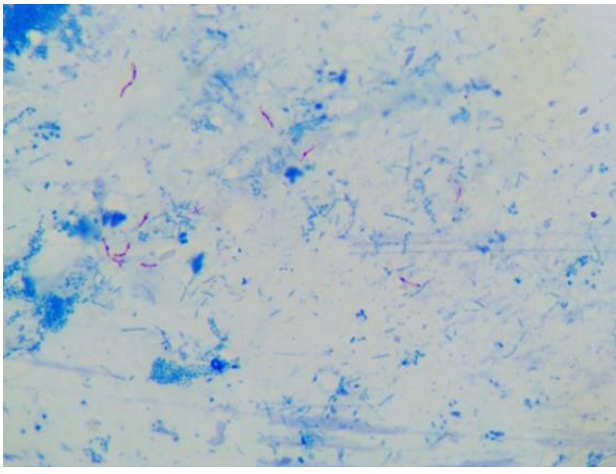

(a)

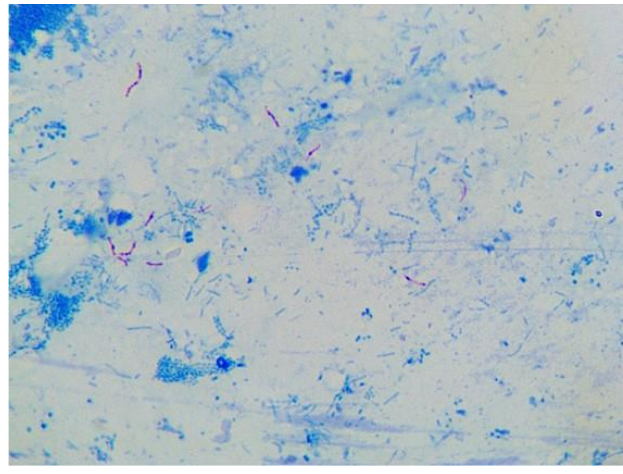

(b)

Figure 3. Image enhancement process: (a) The original RGB image, (b) the enhanced RGB image

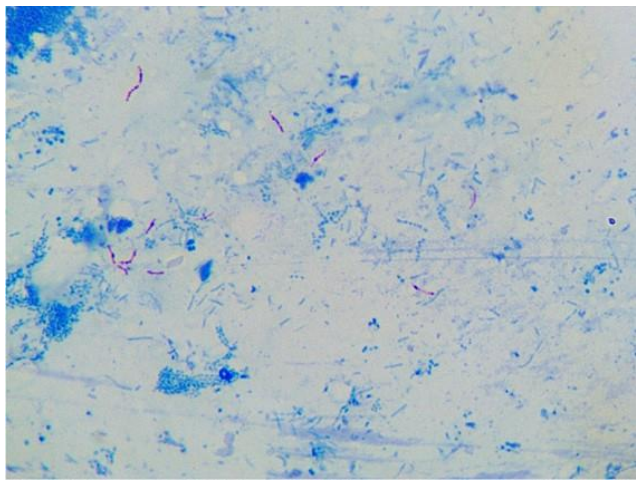

(a)

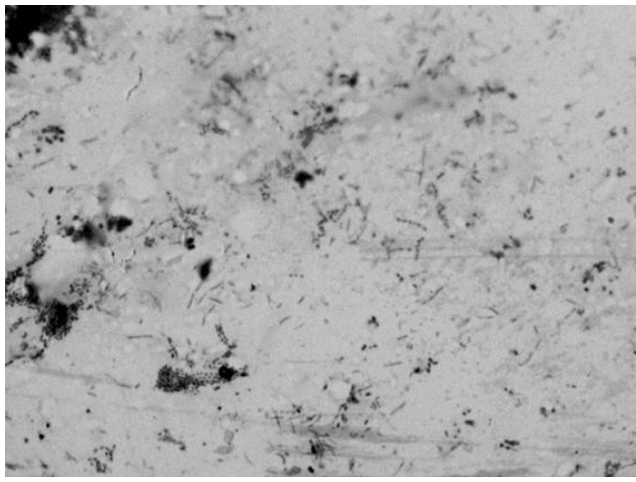

(c)

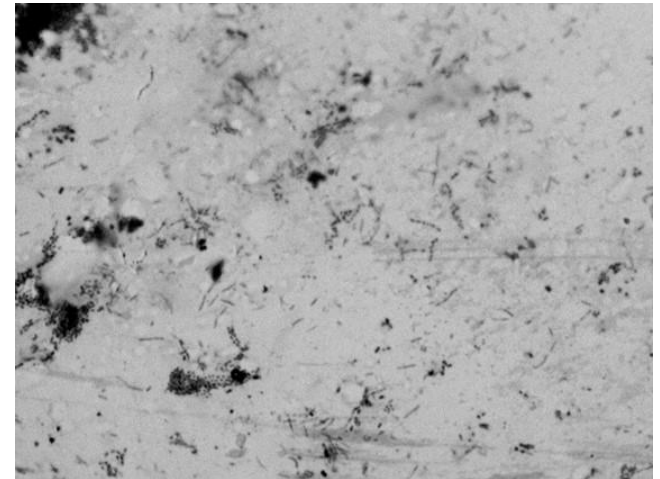

(b)

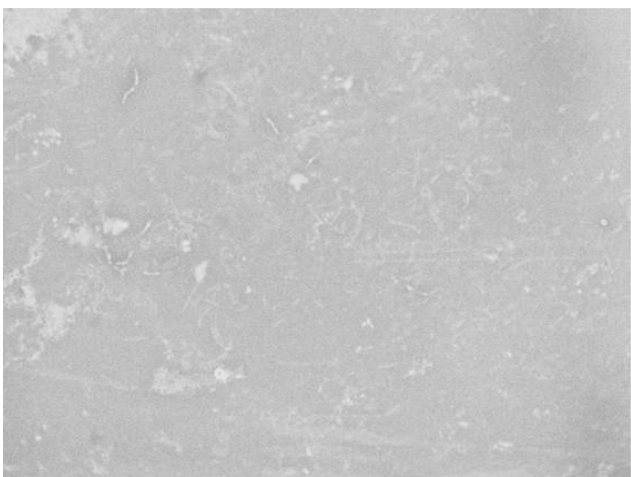

(d)

Figure 4. (a) Enhanced RGB image with primary components $R^{\prime}(x, y), G^{\prime}(x, y)$ and $\mathrm{B}^{\prime}(x, y)$ (b) Red component $R^{\prime}(x, y)$, (c) Green component $\mathrm{G}^{\prime}(x, y)$, (d) Blue component $\mathrm{B}^{\prime}(x, y)$

The transformation functions shown in (8), (9) and (10) are then applied to each component making up enhanced image to highlight the bacilli present in the image. This is shown in Figure 5.

$$
\begin{aligned}
& I M_{1}(x, y)=\left\{\begin{array}{lc}
1.25 \cdot R^{\prime}(x, y) & , \quad 0 \leq R^{\prime}(x, y) \leq 0.8 \\
1 \quad, \quad \text { otherwise }
\end{array}\right.
\end{aligned}
$$

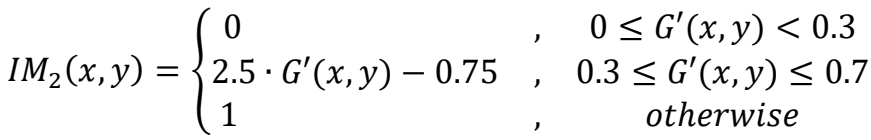

$$
\begin{aligned}
& I M_{3}(x, y)=1 \quad \text { (white image, Fig. 5d) }
\end{aligned}
$$

where $I M_{1}(x, y), I M_{2}(x, y)$ and $I M_{3}(x, y)$, are the components $R, G$ and $B$ respectively. 


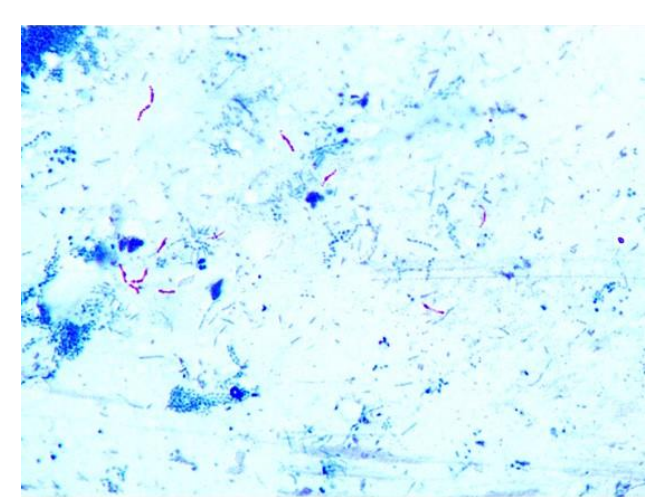

(a)

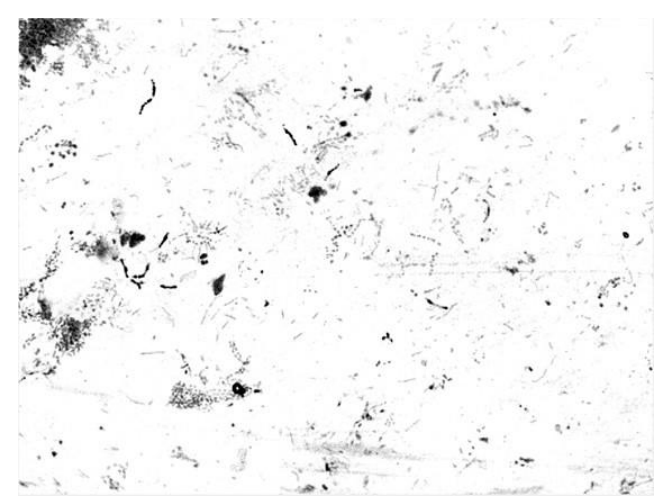

(c)

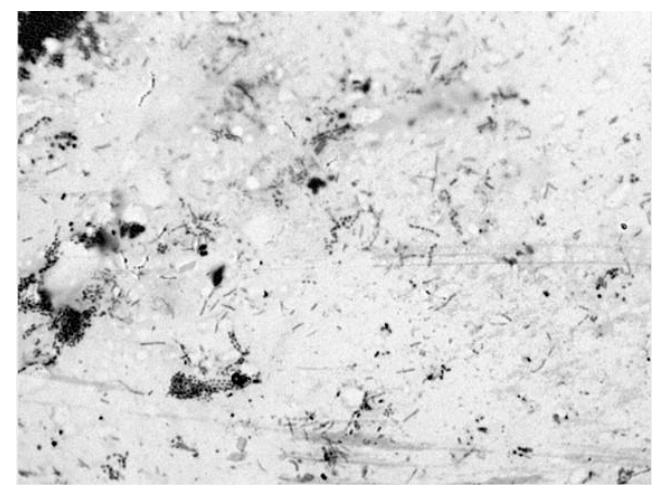

(b)

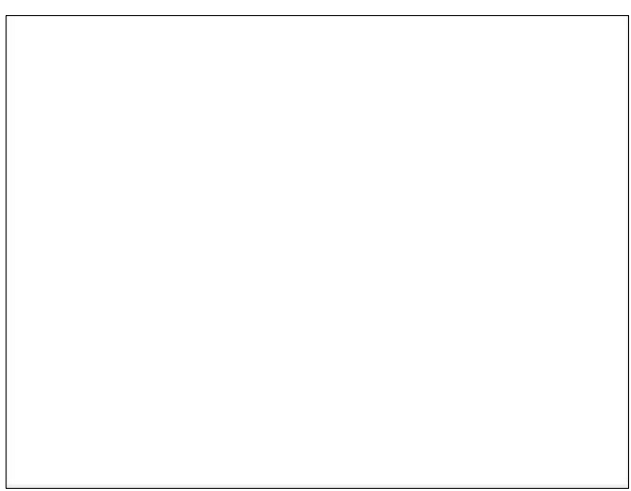

(d)

Figure 5. (a) RGB image with primary components $I M_{1}(x, y), I M_{2}(x, y)$ and $I M_{3}(x, y)$, (b) Red component $I M_{1}(x, y)$, (c) Green component $I M_{2}(x, y)$, (d) Blue component $I M_{3}(x, y)=1$ (white image) .

\subsection{Segmentation}

Once the image has been enhanced, we proceed to the segmentation process. In order to extract the objects of interest (because they are concentrated in the components mentioned above), the improved components $R$ and $G$ are subtracted as expressed in (11). The result of this operation is shown in Figure 6.

$$
I R(x, y)=1.1 \cdot I M_{1}(x, y)-I M_{2}(x, y)
$$

In order to improve the display of all objects in the image, the dynamic range is reduced by applying (12) over the $\operatorname{IR}(x, y)$ image. The result is shown in Figure 7.

$$
I R_{1}(x, y)=\log (1+\operatorname{IR}(x, y))
$$

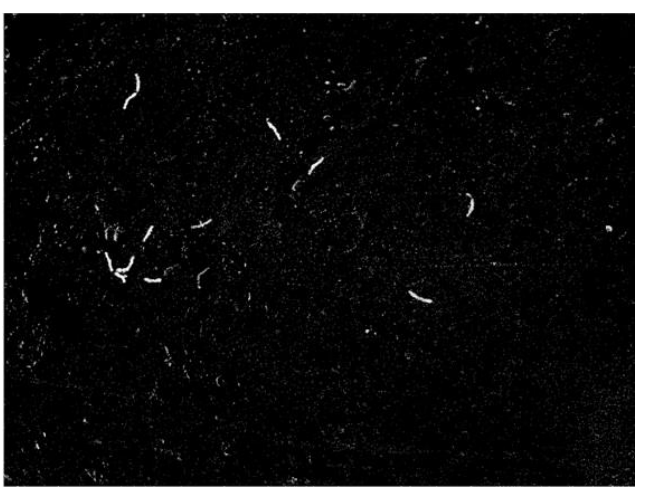

Figure 6. Image obtained by the difference of $I M_{1}(x, y)$ and $I M_{2}(x, y)$

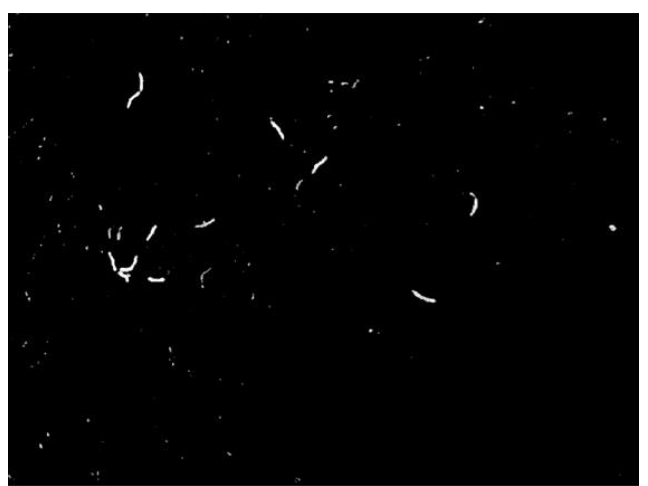

Figure 7. Image $I R_{1}(x, y)$ 
The image $I R_{1}(x, y)$ is filtered with a median filter [17] using a $5 \times 5$ window to standardize the image obtaining $I R_{2}(x, y)$. The result can be seen in Figure 8 . As can be seen, the image has been segmented obtaining the objects of interest and some unwanted artifacts.

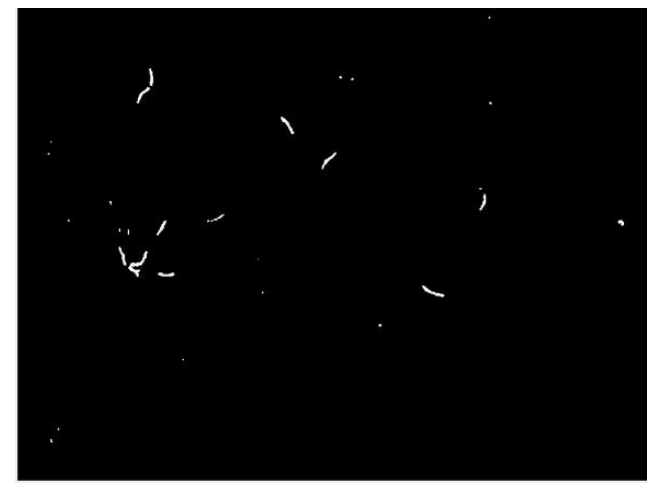

Figure 8. Image $I R_{2}(x, y)$, result of applying median filtering to $I R_{1}(x, y)$

The image obtained is then subjected to a threshold process applying (13). The threshold is fixed and was obtained after performing multiple tests. Figure 9(a) shows an image with a color bar, where the objects of interest are taken with a threshold from 0.75 . The result of the threshold is the $\operatorname{IB}(x, y)$ image shown in Figure 9(b).

$$
I B(x, y)=\left\{\begin{array}{lc}
1 & , \quad I R_{2}(x, y) \geq 0.75 \\
0, & \text { otherwise }
\end{array}\right.
$$

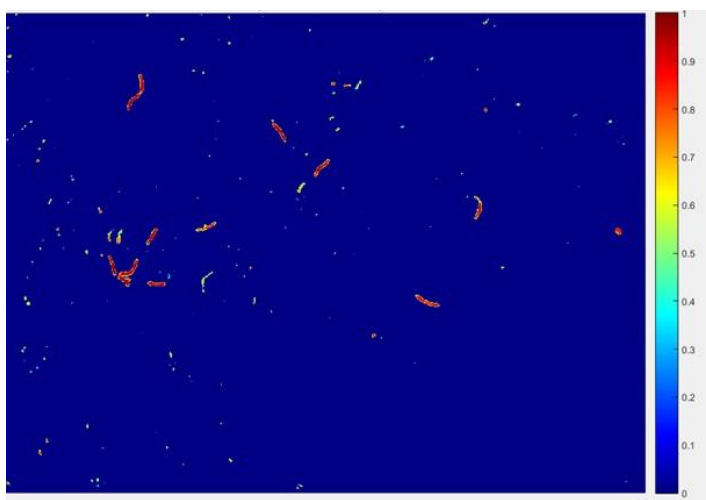

(a)

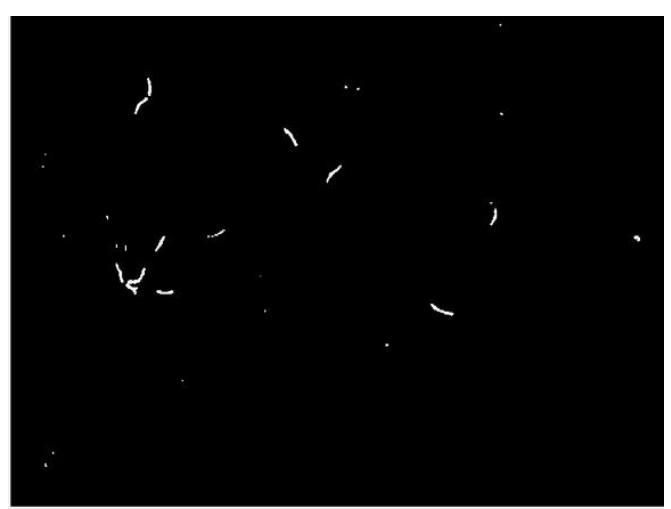

(b)

Figure 9. a) Image Colormap $I R_{2}(x, y)$, b) Binary image $I B(x, y)$

The tagging algorithm [18] is then applied to the $I B(x, y)$ binary image in order to remove tags containing less than 40 pixels; this in order to remove objects that because of their size do not qualify to be bacilli. The result of this process is $I B_{1}(x, y)$. Likewise, in order to give solidity to the obtained objects, the morphological closing process [19] is applied with a structural element EE. This process is indicated in (14).

$$
\begin{aligned}
& \mathbf{I B}_{\mathbf{2}}=\left(\mathbf{I B}_{\mathbf{1}} \oplus \mathbf{E E}\right) \Theta \mathbf{E E} \\
& \mathbf{E E}=\left[\begin{array}{lll}
1 & 1 & 1 \\
1 & 1 & 1 \\
1 & 1 & 1
\end{array}\right]
\end{aligned}
$$


where $\mathbf{I B}_{\mathbf{1}}$ and $\mathbf{I B}_{\mathbf{2}}$ constitute the matrices that represent the images $I B_{1}(x, y)$ and $I B_{2}(x, y)$ respectively. The symbols " $\bigoplus$ " and " $\ominus$ " represent the morphological operations of dilation and erosion respectively. The matrix obtained from this process is $\mathbf{I B}_{\mathbf{2}}$ which represents the image $I B_{2}(x, y)$ and is shown in Figure 10 . The labeling algorithm is applied to the image $I B_{2}(x, y)$, obtaining all the final objects to be able to determine which are possible bacilli. The image of the tagged objects is shown in Figure 11.

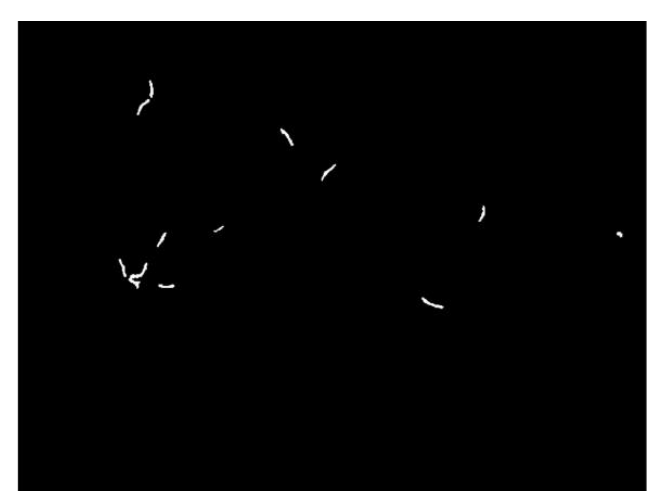

Figure 10. Image $I B_{2}(x, y)$

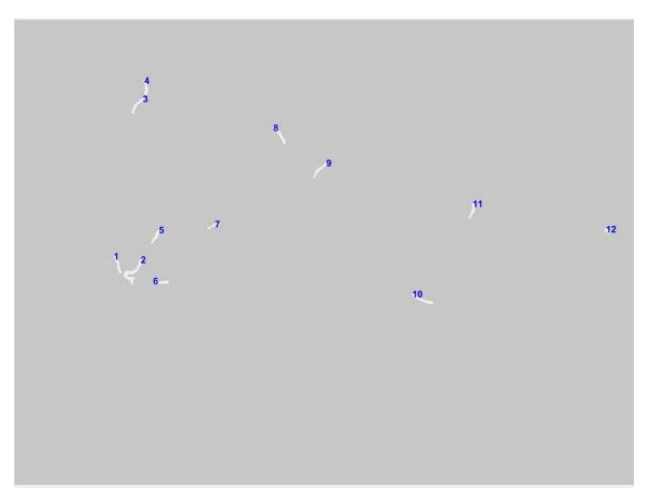

Figure 11. Image of all tagged objects

\subsection{Descriptors calculation}

For the detection process of bacilli in images obtained by ZN bacilloscopy in the different methods, direct, pellet and dilute pellet, the detected objects were analyzed and the geometric and photometric descriptors were calculated.

\subsubsection{Geometric Descriptors}

Four geometric descriptors [20] were calculated for each object obtained from $I B_{2}(x, y)$.

\subsubsection{Area}

The area $A_{n}$, of the nth object $O_{n}(x, y)$ belonging to the binary image $I B_{2}(x, y)$ is equal to the sum of the number of pixels corresponding to the object.

$$
A_{n}=\sum_{x=0}^{M_{n}-1} \sum_{y=0}^{N_{n}-1} O_{n}(x, y)
$$

where $M_{n}$ and $N_{n}$ correspond to the number of rows and columns of the nth object respectively.

\subsubsection{Perimeter}

The perimeter $P_{n}$, of the nth object $O_{n}(x, y)$ belonging to the binary image $I B_{2}(x, y)$ left, right, up or down (4-next-door).

$$
P_{n}=\sum_{x=0}^{M_{n}-1} \sum_{y=0}^{N_{n}-1} O_{n}(x, y), \forall O_{n}(x, y) \in 4-\operatorname{neighbour}\left(O_{n}(x, y)\right)=0
$$

\subsubsection{Eccentricity} is equal.

The eccentricity $E_{n}$, of an ellipse of the nth object $O_{n}(x, y)$ belonging to the binary image $I B_{2}(x, y)$

$$
E_{n}=\sqrt{1-\frac{b_{n}}{a_{n}}}
$$

where $a_{n}$ and $b_{n}$ are the size of the major and minor axis of object $O_{n}(x, y)$ respectively

\subsubsection{Solidity}

The solidity $S_{n}$, of the nth object $O_{n}(x, y)$ belonging to the binary image $I_{2}(x, y)$ is obtained in the following way.

$$
S_{n}=\frac{P_{n}}{C X_{n}}
$$

where $C X_{n}$ is the convex area of the nth object. 


\subsubsection{Photometric Descriptors}

Six photometric descriptors [21] where calculated for each objet obtained from $B_{2}(x, y)$

a. Percentile 35 in the red band (PR35 $)$.

b. Percentile 65 in the green band (PG65n $)$.

c. Standard deviation in the red band $\left(\mathrm{STDR}_{n}\right)$

d. Standard deviation in the green band $\left(\mathrm{STDG}_{n}\right)$

e. Standardized energy in the red band $\left(\mathrm{ER}_{n}\right)$.

f. Standardized energy in the red band $\left(E G_{n}\right)$.

To obtain the a-th percentile $\left(P e_{a, n}\right)$ the histogram [22] of the n-th object $O_{n}(x, y)$ will be defined first.

$$
h_{n}\left(r_{k}\right)=m_{k}
$$

where $r_{k}$ is the k-th intensity level of the object and $m_{k}$ is the number of pixels in the object that has the $r_{k}$ intensity level.

Then the index for the desired percentile is obtained, being $M_{n}$ and $N_{n}$ number of rows and columns respectively of the nth object $O_{n}(x, y)$ that belongs to the image of interest. The value of $i d_{n}$ obtains the number of pixels of the desired percentile.

$$
i d_{n}=\frac{a \cdot M_{n} \cdot N_{n}}{100}, \quad a=1,2, \ldots, 99
$$

Once the number of pixels for this percentile is obtained, the values of the histogram are added until the number of calculated pixels is reached.

$$
i d_{n} \leq \min \left(\sum_{j=0}^{r_{k}} h_{n}\left(r_{j}\right)\right) \quad, \quad r_{k}=0,1,2 \ldots, 255
$$

Therefore, in (22) the percentile a is obtained that is equal to $r_{k}$ that complies with (21).

$$
I_{n}=r_{k}
$$

\subsection{Clasification of objects}

For the classification of the objects, a Support Vector Machine (SVM) was implemented. The SVM finds the hyperplane that maximizes the margin between the TB-positive and TB-negative classes in the characteristics space [23-25]. The SVM dimension used is $K_{p}+1$, where $K_{p}$ is the number of descriptors that the SVM input has for each preparation method. In this case, the dimension is the same for all preparation methods.

The descriptors used are shown in Table 1 and are the same for all preparation methods. With these chosen descriptors the best results were obtained, both in sensitivity and specificity. The SVM input vectors corresponding to each object obtained by each preparation method are defined by (23), (24) and (25) for the direct method, pellet and diluted pellet respectively.

$$
\begin{aligned}
& I_{n 1}=\left[A_{n 1}, P_{n 1}, E_{n 1}, S_{n 1}, \mathrm{PR} 35_{\mathrm{n} 1}, \mathrm{PG}_{\mathrm{n} 1}, \mathrm{STDR}_{n 1}, \mathrm{STDG}_{n 1}, \mathrm{ER}_{n 1}, \mathrm{EG}_{n 1}\right] \\
& I_{n 2}=\left[A_{n 2}, P_{n 2}, E_{n 2}, S_{n 2}, \mathrm{PR} 35_{\mathrm{n} 2}, \mathrm{PG}_{\mathrm{n} 2}, \mathrm{STDR}_{n 2}, \mathrm{STDG}_{n 2}, \mathrm{ER}_{n 2}, \mathrm{EG}_{n 2}\right] \\
& I_{n 3}=\left[A_{n 3}, P_{n 3}, E_{n 3}, S_{n 3}, \mathrm{PR} 35_{\mathrm{n} 3}, \mathrm{PG}_{\mathrm{n} 3}, \mathrm{STDR}_{n 3}, \mathrm{STDG}_{n 3}, \mathrm{ER}_{n 3}, \mathrm{EG}_{n 3}\right]
\end{aligned}
$$

Table 1 shows the number of vectors used to train SVM for each preparation method, and the number of vectors for validation purposes. For the implementation of the SVM a kernel was applied, because the data were not linearly separable. For all preparation methods a grade 6 polynomial was used. In (26) and (27) the following operation is shown.

$$
K_{p}\left(I_{n p}, s v_{p}\right)=\left(I_{n p}^{T} \cdot s v_{p}+1\right)^{6}
$$

where $s v_{p}$ is the SVM support vector for each $p$ separation method.

$$
y_{n p}=\sum \alpha_{p} \cdot K_{p}\left(I_{n p}, s v_{p}\right)+b
$$

where $\alpha_{p}$ is the Lagrange multiplier and has the same dimensions as its corresponding support vector and $b$ is the bias. Figures 12-14 show how SVM classifies objects in images from the different preparation methods. 
Table 1. SVM configuration

\begin{tabular}{cccc}
\hline Descriptor / Method & SVM Direct & SVM Pellet & SVM Diluted Pellet \\
\hline$A_{n p}$ & $\mathrm{x}$ & $\mathrm{x}$ & $\mathrm{x}$ \\
$P_{n p}$ & $\mathrm{x}$ & $\mathrm{x}$ & $\mathrm{x}$ \\
$e_{n p}$ & $\mathrm{x}$ & $\mathrm{x}$ & $\mathrm{x}$ \\
$S_{n p}$ & $\mathrm{x}$ & $\mathrm{x}$ & $\mathrm{x}$ \\
$\mathrm{PR} 35_{\mathrm{np}}$ & $\mathrm{x}$ & $\mathrm{x}$ & $\mathrm{x}$ \\
$\mathrm{PG}_{\mathrm{np}}$ & $\mathrm{x}$ & $\mathrm{x}$ & $\mathrm{x}$ \\
$\mathrm{STDR}_{n p}$ & $\mathrm{x}$ & $\mathrm{x}$ & $\mathrm{x}$ \\
$\mathrm{STDG}_{n p}$ & $\mathrm{x}$ & $\mathrm{x}$ & $\mathrm{x}$ \\
$\mathrm{ER}_{n p}$ & $\mathrm{x}$ & $\mathrm{x}$ & $\mathrm{x}$ \\
$E G_{n p}$ & x & Polynomial order 6 & Polynomial order 6
\end{tabular}

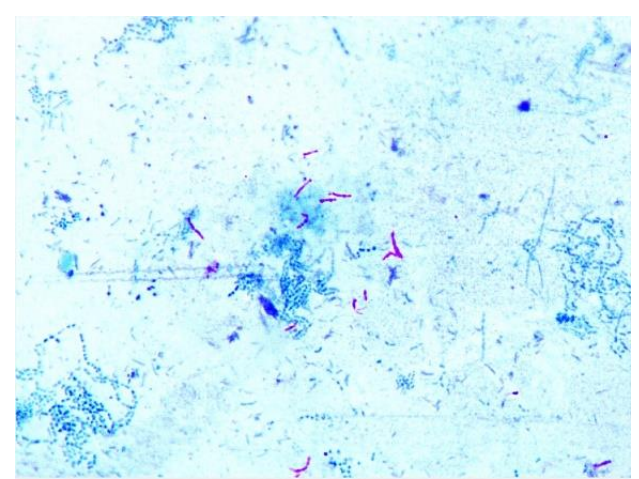

(a)

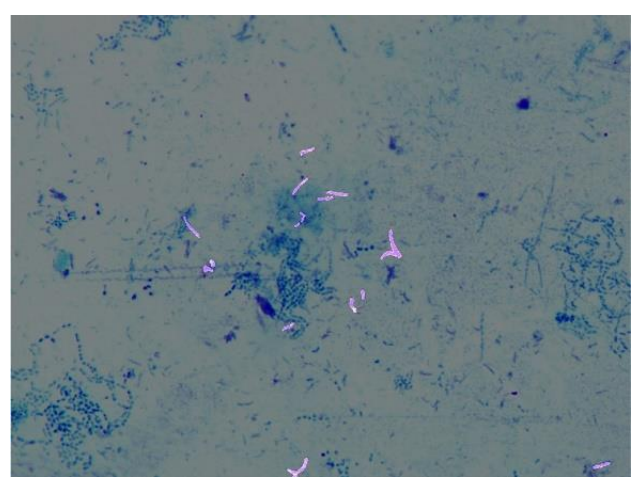

(b)

Figure 12. Classification at object level for the direct method

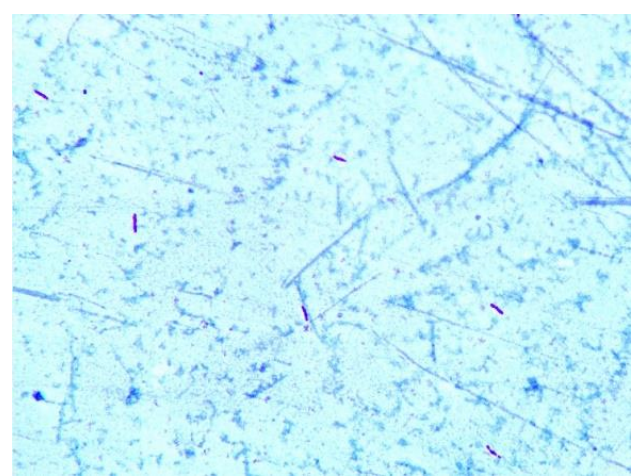

(a)

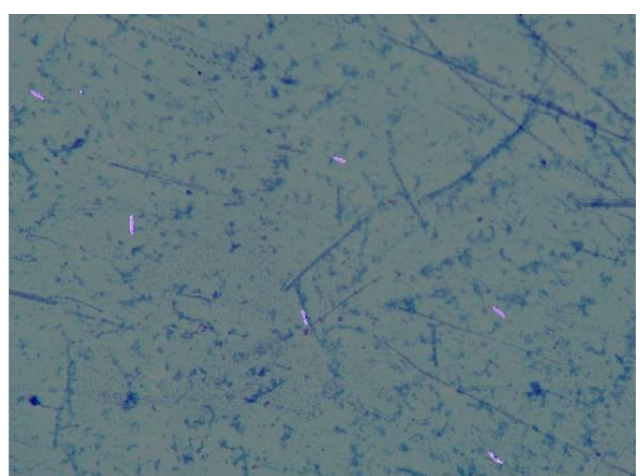

(b)

Figure 13. Object level classification for the pellet method

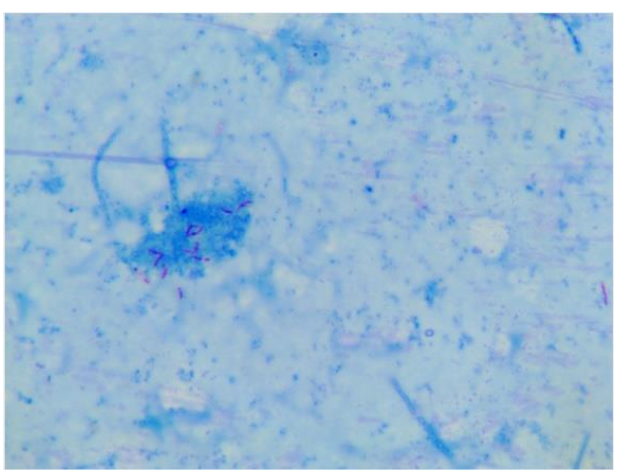

(a)

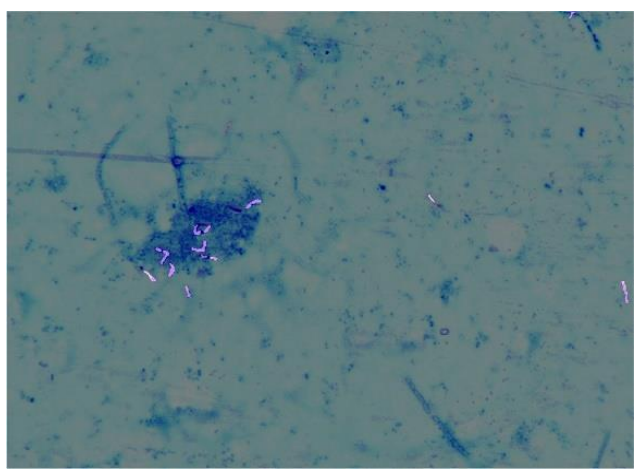

(b)

Figure 14. Object level classification for the diluted pellet method 


\section{RESULTS}

For the present work, $225 \mathrm{ZN}$ paving images, 75 images by the direct method, 75 images by the pellet method and 75 images by the diluted pellet method were analyzed. All images were obtained from different patients. Sample preparation is a standardized process and this is indicated in [26]. In order to validate, we worked with more than 350 objects between bacilli and non-bacilli obtained from the different processed images. Table 2 shows the distribution of the data.

Tabla 2. Data distribution

\begin{tabular}{cccc}
\hline Descriptor / Method & Direct & Pellet & Diluted Pellet \\
\hline Data Total & 769 & 751 & 735 \\
Training Data & 538 & 526 & 515 \\
Descriptors & 10 & 10 & 10 \\
Data Validation & 231 & 225 & 220 \\
\hline
\end{tabular}

Sensitivity and specificity values were obtained from (28) and (29).

$$
\begin{aligned}
& \text { sensibility }=\frac{V P}{V P+F N} \cdot 100 \\
& \text { specificity }=\frac{V N}{V N+F P} \cdot 100
\end{aligned}
$$

where VP stands for True Positive and the correctly classified positive bacilli. Objects recognized by the specialist as bacilli and that match the software; VN stands for True Negative and are the correctly classified negative bacilli. Objects detected by the software as NO bacilli and coinciding with the specialist; FP stands for False Positive and are negative bacilli classified as positive. Objects that the software detects as bacilli and that are NOT bacilli; and FN that means False Negative that are the positive bacilli classified as negative. Bacilli that are not detected by the software as bacilli, but have been recognized and accepted by the specialist.

Two medical technologists from the Tuberculosis Laboratory of the Universidad Peruana Cayetano Heredia, both with extensive experience in the preparation, analysis and diagnosis of TB, participated in the validation of the proposed method. The specialists evaluated separately all the images that were submitted to the proposed algorithm (without knowing the previous results of the software).

Tables 3-5 show the results obtained for sensitivity and specificity. Specialist No. 1 is denoted as E1, specialist No. 2 as E2, E1 or E2 as E3 and E1 and E2 as E4. Figure 15 shows the best results obtained for sensitivity and specificity from Tables 3-5.

Table 3. Sensitivity and specificity analysis by the direct method

\begin{tabular}{ccccc}
\hline $\begin{array}{l}\text { Analysis / } \\
\text { Specialist }\end{array}$ & E1 & E2 & E3 & E4 \\
\hline Sensitivity & $92.55 \%$ & $93.25 \%$ & $92.17 \%$ & $93.67 \%$ \\
Specificity & $84.29 \%$ & $88.24 \%$ & $89.23 \%$ & $83.56 \%$ \\
\hline
\end{tabular}

Table 4. Sensitivity and specificity analysis by pellet method

\begin{tabular}{ccccc}
\hline $\begin{array}{l}\text { Analysis / } \\
\text { Specialist }\end{array}$ & E1 & E2 & E3 & E4 \\
\hline Sensitivity & 91.14 & 91.08 & 90.15 & 92.13 \\
Specificity & 80.49 & 79.52 & 82.58 & 77.71 \\
\hline
\end{tabular}

Table 5. Sensitivity and specificity analysis by diluted pellet method

\begin{tabular}{ccccc}
\hline $\begin{array}{l}\text { Analysis / } \\
\text { Specialist }\end{array}$ & E1 & E2 & E3 & E4 \\
\hline Sensitivity & 92.31 & 92.31 & 91.82 & 92.81 \\
Specificity & 81.25 & 81.25 & 83.61 & 79.10 \\
\hline
\end{tabular}

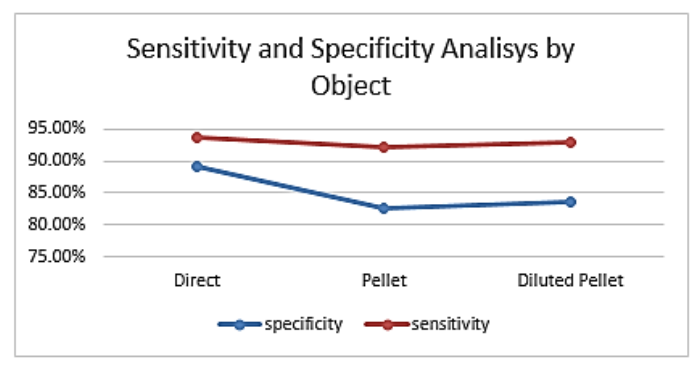

Figure 15 . Sensitivity and specificity analisys by object 
It must be taken into account that the images obtained in any of the three preparation methods must be well focused so as not to cause error. Likewise, it is important that the samples are correctly prepared, that is to say, that there is no leakage of the fuchsin dye on the image, as well as the bacilli must be well marked with this dye to improve the sensitivity and specificity, and also avoid that a bacillus is split and can affect the counting of these.

For the description of the objects considered as bacilli, both shape and photometric descriptors were taken into account, and specifically that comply with fuchsin staining, because of this the 35 th and 65 th percentiles were used in the $R$ and $G$ components of the image respectively, given that component $B$ was set to 1 . The best result in sensitivity is obtained in the direct method, however, all three methods exceed $90 \%$ sensitivity. Likewise, the best specificity result is achieved in direct method, almost $90 \%$, and the lowest index in pellet method. This is because there are objects that without being bacilli can be marked by fuchsin product of the sample preparation process. The most common method of preparation, in any health center, is the direct method.

\section{ACKNOWLEDGMENTS}

The present work was carried out thanks to the research funds of the "Fondo Nacional de Desarrollo Científico, Tecnológico y de Innovación Tecnológica (FONDECYT)", an initiative of the "Consejo Nacional de Ciencia, Tecnología e Innovación Tecnológica (CONCYTEC)”, (no. 1473-2014- C1).

\section{REFERENCES}

[1] K. R. Steingart, et al., "Fluorescence versus conventional sputum smear microscopy for tuberculosis: a systematic review." Lancet Infect. Dis., vol/issue: 6(9), pp. 570-581, 2006.

[2] O. A. Osibote, et al., "Automated focusing in bright-field microscopy for tuberculosis detection," J. Microsc., vol/issue: 240(2), pp. 155-163, 2010.

[3] N. A. Ismail, et al., "Performance of a Novel Algorithm Using Automated Digital Microscopy for Diagnosing Tuberculosis," American Journal of Respiratory and Critical Care Medicine, vol. 191, pp. 1443-1449, 2015.

[4] I. C. Chang, et al., "Physicians acceptance of pharmacokinetics-based clinical decision support systems," Expert Syst. Appl., vol/issue: 33(2), pp. 296-303, 2007.

[5] M. G. Costa, et al., "Automatic identification of Mycobacterium tuberculosis with conventional light microscopy," Proc. of 30th Annual International IEEE Eng Med Biol Soc. Vancouver, Canada, pp. 382-385, 2008.

[6] P. Sadaphal, et al., "Image processing techniques for identifying Mycobacterium tuberculosis in ziehl-neelsen stains," International Journal of Tuberculosis and Lung Disease, vol/issue: 12(5), pp. 579-582, 2008.

[7] M. K. Osman, et al., "Tuberculosis bacilli detection in Ziehl-Neelsen- stained tissue using affine moment invariants and Extreme Learning Machine," Proc. of IEEE 7th International Colloquium on Signal Processing and its Applications (CSPA), pp. 232-236, 2011.

[8] M. K. Osman, et al., "A genetic algorithm-neural network approach for Mycobacterium tuberculosis detection in Ziehl-Neelsen stained tissue slide images," Proc. of 10th International Conference on Intelligent Systems Design and Applications (ISDA), pp. 1229-1234, 2010.

[9] M. K. Osman, et al., "Compact single hidden layer feed forward network forMycobacterium tuberculosis detection," Proc. of IEEE International Conference on Control System, Computing and Engineering (ICCSCE), 432-436, 2011.

[10] Y. Zhai, et al., "Automatic Identification of Mycobacterium tuberculosis from ZN-stained sputum smear: Algorithm and system design," Proc. of IEEE International Conference on Robotics and Biomimetics (ROBIO), Tianjin, pp. 41-46, 2010.

[11] C. F. F. C. Filho, et al., "Mycobacterium tuberculosis Recognition with Conventional Microscopy," Proc. IEEE Eng Med Biol Soc., pp. 6263-8, 2012.

[12] M. G. Costa, et al., "A sputum smear microscopy image database for automatic bacilli detection in conventional microscopy," Proc. of 36th Annual International Conference of IEEE Engineering in Medicine and Biology Society (EMBC), pp. 2841-2844, 2014.

[13] S. Ayas and M. Ekinci, "Random forest-based tuberculosis bacteria classification in images of ZN-stained sputum smear samples." SIViP, vol/issue: 8(1), pp. 49-61, 2014.

[14] R. O. Panicker, et al., "Automatic detection of tuberculosis bacilli from microscopic sputum smear images using deep learning methods," International Journal of Biocybernetics and Biomedical Engineering, vol/issue: 38(8), pp. 691699, 2018.

[15] A. R. Robertson, "The CIE 1976 Color-Difference Formulae," Color Research \& application, vol/issue: 2(1), pp. 7-11, 1977.

[16] A. Jain, "Fundamentals of digital image processing," NJ, Prentice-Hall, 1988.

[17] W. K. Pratt, "Median filtering," Semiannual Report, Image Processing Institute, Univ. of Southern California, pp. 116-123, 1975.

[18] H. Lifeng, et al., "The connected-component labeling problem: A review of state-of-the-art algorithms," Pattern Recognition, vol. 70, pp. 25-43, 2017.

[19] G. J. F. Banon, et al., "Mathematical Morphology and its Applications to Signal and Image Processing," Proceedings of the 8th international symposium on mathematical morphology (ISMM'07), 2007.

An algorithm for detection of Tuberculosis bacilli in Ziehl-Neelsen sputum smear ... (Christian del Carpio) 
[20] D. Zhang and G. Lu, "Review of shape representation and description techniques," Pattern Recognition, vol/issue: 37(1), pp. 1-19, 2004.

[21] R. Khan, et al., "Discriminative color descriptors," CVPR, pp. 2866-2873, 2013.

[22] R. Gonzalez and R. Woods, "Digital Image Processing," Second Edition, Pearson Education, 2002.

[23] C. C. Chang and C. J. Lin, "LIBSVM: A Library for Support Vector Machines," ACM Trans. on Intell Sys and Tech, vol/issue: 2(3), pp. 21-27, 2011.

[24] C. Cortes and V. Vapnik, "Support-vector networks," Mach Learn, vol/issue: 20(3), pp. 273-297, 1995.

[25] F. Vilariño and E. Valveny, "Clasificador SVM (Support Vector Machine)," 2018. Available: https://www.coursera.org/learn/deteccion-objetos/lecture/iGdzT/14-5-support-vector-machines-svm-conceptosbasicos).

[26] Organización Panamericana de la Salud, "Manual para el diagnóstico bacteriológico de la tuberculosis,” 2009.

\section{BIOGRAPHIES OF AUTHORS}

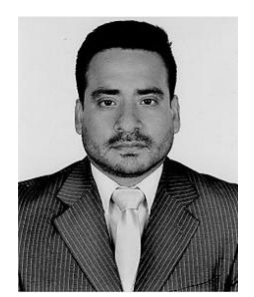

Christian del Carpio, he received the B.S. degree in Electrical Engineering in 2005 from the San Martin de Porres Private University (USMP), Lima, Peru. In the year 2015, he obtained a Master degree in Science, from National University of Engineering (UNI), Peru. Currently, he is working as a research professor for the undergraduate program of the schools of Electrical Engineering from the San Martin de Porres University (USMP), Lima, Peru, and the Peruvian University of Applied Sciences (UPC), Lima, Peru. He is working as a research professor for the undergraduate and postgraduate programs of the schools of Electrical Engineering and the school of Mechatronics Engineering from the National University of Engineering (UNI), Lima, Peru. His research interests include image and signal processing.

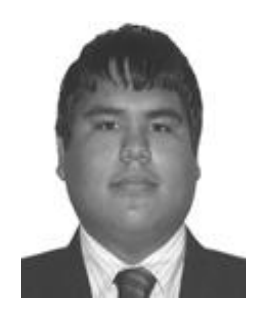

Erwin Dianderas, Electronic Engineer of the Universidad Peruana de Ciencias Aplicadas (UPC), M.Sc(c) Telecommunications from the Universidad Nacional de Ingenieria. He currently works on tuberculosis research, developing tools to assist in its detection.

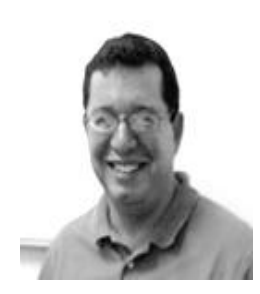

Mirko Zimic, PhD degree in Control and Prevention of Diseases from Johns Hopkins University, MSc. Biochemistry from the Peruvian University Cayetano Heredia (UPCH) and also obtained his BSc. Physics at the National University of Engineering. Currently, he is the head of the Laboratory of Bioinformatics and Molecular Biology of the UPCH and leads a multidisciplinary working group, with whom he researches in different areas of science and technology.

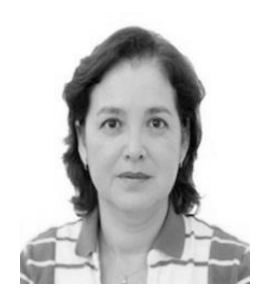

Patricia Sheen, Biologist, Doctorate in Disease Control from Johns Hopkins University, MSc. Biochemistry from Universidad Peruana Cayetano Herediaand BSc. Biochemistry from Universidad Ricardo Palma. Currently, she is Coordinator of the Infectious Diseases Research Laboratory (LID) of the Universidad Peruana Cayetano Heredia (UPCH). The research group which she leads together with PhD Mirko Zimic has as its line of research to understand the mechanism of resistance to pyrazinamide in Mycobacterium tuberculosis.

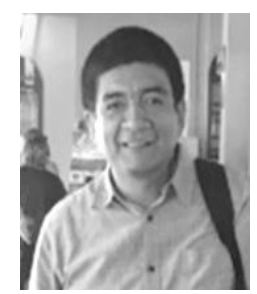

Jorge Coronel, Medical Technologist in the specialty of Clinical Laboratory, with degree of MSc in Microbiology carried out in the Universidad Nacional Mayor de San Marcos and BSc in Medical Technology in the Universidad Peruana Cayetano Heredia (UPCH). Currently, he is the Coordinator of the Mycobacteria Laboratory at the Research and Development Laboratory (LID) of UPCH. He has developed research especially in the area of Clinical Microbiology and topics related to antimicrobial resistance especially in tuberculosis as a Public Health problem. 


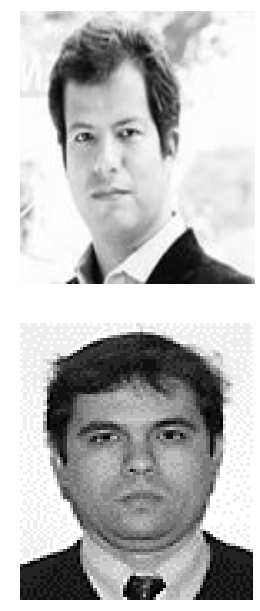

Roberto Lavarello, Associate Professor of Electronic Engineering at the Pontificia Universidad Católica del Perú (PUCP), he obtained his Bachelor of Science and Engineering degrees from the PUCP and his Master and Doctor of Electrical and Computer Engineering from the University of Illinois at Urbana-Champaign (UIUC, USA). He has been a Fulbright Scholar, mentor of NASA's MUST program, and is currently a mentor of the IEEE Engineering in Medicine and Biology Society (IEEE EMBS). Dr. Lavarello currently serves as Head of Research at the PUCP Medical Imaging Laboratory and Director of the PUCP Master's Degree in Signal Processing and Digital Imaging.

Guillermo Kemper, received the B.S. degree in Electrical Engineering in 1994 from the Antenor Orrego Private University (UPAO), Trujillo, Peru. In years 1996 and 2001, he obtained a Master degree in Electrical Engineering and a $\mathrm{PhD}$ in Electrical and Communications Engineering, respectively, from the State University of Campinas (UNICAMP), Brazil. He has taken part for more than 3 years in the $\mathrm{CPqD}$ research agreement between the Brazilian Telecommunications Company Telebras and UNICAMP, developing and designing audio and video coders. Currently, he is working as a research professor for the undergraduate and postgraduate programs of the schools of Electrical Engineering of the San Martin de Porres University (USMP) and the Peruvian University of Applied Sciences (UPC), both located in Lima, Peru. In addition, he is a researcher at the National Institute of Research and Training in Telecommunications (INICTEL-UNI), Lima, Peru. His research interests include voice, audio, images and video processing, digital communications and digital television. 